\title{
Guidelines for Rational Cancer Therapeutics
}

\author{
Byunghee Yoo ${ }^{1}$, Ann-Marie Billig ${ }^{2}$ and Zdravka Medarova ${ }^{1 *}$ \\ ${ }^{1}$ MGH/MIT/HMS Athinoula A. Martinos Center for Biomedical Imaging, Massachusetts General Hospital and Harvard \\ Medical School, Boston, MA, United States, ${ }^{2}$ Bouvé College of Health Sciences, Northeastern University, Boston, MA, \\ United States
}

Traditionally, cancer therapy has relied on surgery, radiation therapy, and chemotherapy. In recent years, these interventions have become increasingly replaced or complemented by more targeted approaches that are informed by a deeper understanding of the underlying biology. Still, the implementation of fully rational patient-specific drug design appears to be years away. Here, we present a vision of rational drug design for cancer that is defined by two major components: modularity and image guidance. We suggest that modularity can be achieved by combining a nanocarrier and an oligonucleotide component into the therapeutic. Image guidance can be incorporated into the nanocarrier component by labeling with a specific imaging reporter, such as a radionuclide or contrast agent for magnetic resonance imaging. While limited by the need for

OPEN ACCESS

Edited by:

Zaver Bhujwalla,

Johns Hopkins University,

United States

Reviewed by:

Aslam Khan,

University of Missouri, United States

Rossella Canese,

Istituto Superiore di Sanità, Italy

${ }^{*}$ Correspondence:

Zdravka Medarova

zmedarova@partners.org

Specialty section:

This article was submitted to Cancer Imaging and Diagnosis,

a section of the journal

Frontiers in Oncology

Received: 04 October 2017 Accepted: 29 November 2017 Published: 12 December 2017

Citation:

Yoo B, Billig A-M and Medarova Z (2017) Guidelines for Rational Cancer Therapeutics. Front. Oncol. 7:310. doi: 10.3389/fonc.2017.00310 additional technological advancement in the areas of cancer biology, nanotechnology, and imaging, this vision for the future of cancer therapy can be used as a guide to future research endeavors.

Keywords: imaging, cancer, therapy, rational, nanomedicine

\section{INTRODUCTION}

The recent past has seen an impetus to develop more personalized therapies for cancer, as part of the general concept of precision medicine. In this review, we look at a component of precision medicine focused on the specific design of the therapeutic. We suggest that it is possible to design therapeutics that are fully rational. Namely, we envision rational therapeutics as therapeutics based entirely on predesigned components. This strategy is different than strategies that we have seen in the past or are seeing currently. Traditional approaches are largely reliant on trial-and-error discovery. A prime example is penicillin, which was discovered by pure chance. Even more rational modern approaches such as combinatorial screening, have a large element of trial-and-error in their discovery. The approach we are talking about has no trial-and-error components. It is entirely predesigned, meaning that that each patient will be given a treatment plan that would have been determined as nearly $100 \%$ effective because of their genetic makeup, anatomy, and physiology. Because of that, this approach fits perfectly into the concept of precision medicine-if an approach is truly rational, it can tell us whether a patient is going to respond to treatment before that patient has even been injected with the drug.

Key components of rational therapeutics are modularity and image guidance. Modularity allows us to synthesize libraries of therapeutic agents that are optimized for a given indication or a given patient in terms of size, surface coating and charge, hydrophilicity/hydrophobicity; antigentargeting through incorporation of targeting peptides, and therapeutic moiety. Image guidance answers questions about the individual biology of a given patient or indication. It is necessary 
because we know that each organ and each patient are highly variable in terms of vascularity, fat content, vascular permeability, inflammatory profile, etc. Because of these differences, we need to vary features such as physicochemical drug design, injection dose, schedule, and even route, in order to provide the best and most effective treatment possible for every individual patient. Imaging is the only modality that can provide answers to these questions.

In our experience, an ideal candidate for rational drug design has three components: a DNA/RNA therapeutic component, a nanoparticle carrier, and an imaging reporter. DNA/RNAtargeted methods could be an integral part of rational therapeutics. This essential component takes advantage of the "coded" nature of the genome and transcriptome. Because of that, DNA/ RNA-targeted methods provide an ideal platform for completely rational design of diagnostic and therapeutic agents based on the phenomenon of complementarity. This approach can be used while relying on recent advances in genome sequencing like antisense oligonucleotides [locked nucleic acid (LNA) oligos, antagomirs, miRNA sponges], small interfering RNA (siRNA) duplexes, or ribozymes. These molecules can be synthesized to target portions of the code that are aberrant in disease and thus the unique genome of the patient would in turn direct us to an equally unique cocktail of therapeutic agents. This constitutes a prime example of achievable individualized medicine.

Nanotechnology is another component of the envisioned rational therapeutic. Specifically, nanoparticles are carriers that can incorporate all three components of the drugs that we envision. They can easily be functionalized with oligonucleotides (antisense, siRNA, etc.) without interfering with the functionality of the oligos. Their design can be fine-tuned using standard synthetic chemistry, e.g., liposomes, iron oxide nanoparticles, etc. Finally, they can be labeled with an imaging reporter, e.g., radionuclide. An example is presented by polymer-coated iron oxide nanoparticles. By varying the ratios of coating to iron, the size of the nanoparticles can be adjusted. In addition, fairly standardized chemistry can be used to incorporate different coats onto the nanoparticles, e.g., dextran, carboxydextran, starch, polyethylene glycol (PEG), etc. These changes in size and surface coating result in very diverse biological fates, related to the agents' pharmacokinetics and pharmacodynamics.

Image guidance is the third component of the envisioned drug design. By labeling the drug with an imaging reporter, we can monitor the delivery of the therapeutic agent to the tissue of interest. Image-guided delivery can be instrumental to assess and control delivery to the target tissues. Imaging can be used to determine the optimal drug design, delivery schedule, route, and therapeutic dose on an individual basis and to suggest alternatives should therapy fail in a given patient. In our view, dual-modality approaches, such as PET-MR or PET-CT would be optimal. PET-MR specifically could obtain near perfect spatial registration of molecular/functional positron emission tomography (PET) and anatomic/functional magnetic resonance imaging (MRI). This combination allows highly detailed anatomical images (MR) to be co-registered with PET images that have greater sensitivity and the capability for precise quantitation of local drug concentration.

\section{OLIGONUCLEOTIDE DESIGN}

Of the three elements of rational therapeutics, oligonucleotide development is by far the most advanced. Partly, the reason for this investment in the development of oligonucleotide therapeutics stems from the vast potential to address therapeutic challenges, including undruggable targets. Synthetic oligonucleotides include, among others, antisense oligonucleotides, mRNA oligos, siRNA, microRNA inhibitors or mimics, and more recently, long non-coding RNA modulators. Unlike biologics or small molecules, the listed synthetic oligonucleotides have the advantage of generally binding their targets through direct Watson-Crick complementarity. This fundamentally straightforward property provides an opportunity for rational, computational design of therapeutics, based on the simple knowledge of the target and its sequence.

Nevertheless, there is a list of challenges related to the design of synthetic oligonucleotides for therapy. These challenges relate to the fact that the chemical architecture of the oligonucleotide needs to be fine-tuned according to the requirements for favorable pharmacokinetics. This includes ADME, long-term stability, safety, and immunogenicity, and capacity to engage the target with high affinity and specificity without off-target effects. The latter is especially important when the oligonucleotide functions in the context of a multi-enzyme complex, as is the case for siRNA.

On the level of oligonucleotide architecture, these issues are addressed by rationally designed chemical modifications. Backbone and sugar modifications are two general strategies that have found application. The most commonly encountered backbone modification is the phosphorothioate linkage. The phosphorothioate (PS) linkage imparts significant resistance to nuclease degradation. However, in addition to this role, the PS linkage also has a major impact on oligonucleotide trafficking and uptake. Specifically, phosphorothioates can promote oligonucleotide binding to serum albumin (1) and effectively alter the pharmacokinetics and circulation half-life of the oligos. Phosphorothioate linkages also enhance the cellular uptake of oligos without a carrier for transfection (2). Finally, phosphorothioate linkages can have a reduced binding affinity for the target (3), necessitating further optimization to offset this effect and increase potency.

Such a level of flexibility can be achieved through sugar modifications. These include, among others: incorporation of $2^{\prime}-\mathrm{O}-\mathrm{Me}$ units to increase affinity and nuclease stability $(4,5)$; the incorporation of LNA analogs $(6,7)$ to dramatically increase binding affinity, improve nuclease stability, and reduce immunogenicity; and the incorporation of a $2^{\prime}-O-M O E$ [2'-O-(2-methoxyethyl)] modification to increase affinity and nuclease stability (8). A thorough review on the subject can be found in Ref. $(8,9)$.

Iterative computational methods are available to arrive at a candidate that has the required properties for a given target. Still, an element of empiricism can be involved, for example, when designing siRNA oligos to an mRNA of interest due to variability in the properties of the specific binding site on the mRNA oligo. Oftentimes, this is addressed by utilizing a cocktail of siRNAs.

Nevertheless, compared to methodologies for the design and selection of small molecule drugs or biologics, the degree 
of empiricism is somewhat limited, and originates from the fact that even short oligos have unique structures that impact their biophysical properties. In this context, we would like to highlight a recent publication, which reported on the development of an automated methodology for the prediction of the pharmacological properties of short DNA/LNA oligonucleotides. Namely, the authors apply quantum mechanical calculations to predict structures and electrostatic surface potentials for the oligos, which are primary determinants of interaction between molecules (10).

As an example, the authors studied the effects of changed internucleoside linkages from a phosphodiester (PO) to a phosphorothioate (PS). LNA-PO-AAG (Figure 1A) was compared with LNA-PS-AAG (with the PSs in the RS configuration) (Figure 1B). The LNA-PS-AAG (RS) modification resulted in a large potential change and exhibited a more scattered topology compared to LNA-PO-AAG, with a shift of the electrostatic potential toward the $5^{\prime}$ end. PS modification also induced changes in the localization of the frontier orbitals. The highest occupied molecular orbitals/lowest unoccupied molecular orbitals are split between adenine and guanine for LNA-PO-AAG (Figure 1A) but the LUMO resides on the central adenines for PS (Figure 1B) (10). The study illustrates the application of quantum mechanical modeling that can be used to understand antisense oligonucleotide properties and to explain the observation that small structural changes in oligonucleotide composition may lead to dramatic shifts in phenotypes, i.e., toxicity, protein binding, and tissue and cell uptake. This type of analysis could be applied in future oligonucleotide drug discovery and would allow the production improved antisense drugs.

\section{NANOCARRIER DESIGN}

Despite the advancements in oligonucleotide design for therapy, rapid entry into the clinic is hampered partly by obstacles related to oligo delivery in vivo. After intravascular administration, oligos get aggregated by serum proteins in the plasma, and/or are rapidly eliminated by the kidneys, resulting in very short
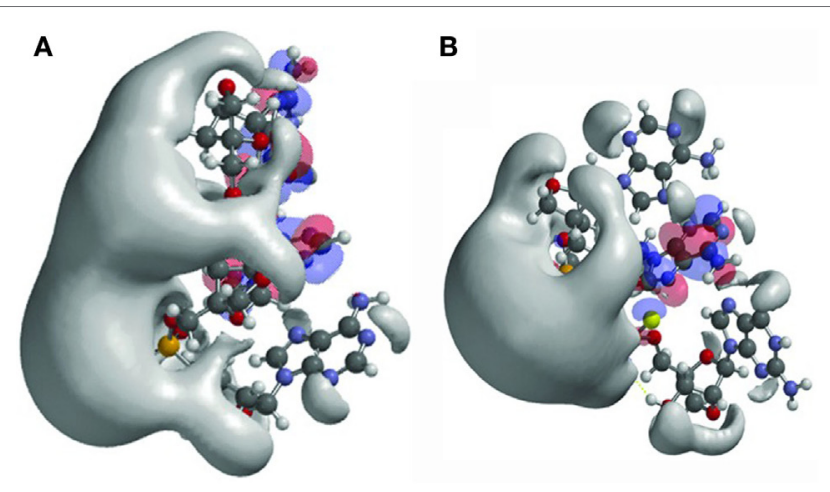

FIGURE 1 | Structures and frontier orbitals (red/blue) for (A) locked nucleic acid (LNA) with the sequence 5'-AAG-3' (5' end at bottom of structure) and (B) LNA with sequence 5'-AAG-3', chemically modified by phosphorothioate internucleoside linkages (PS). The electrostatic potential (negative) is shown in gray and represented by an $/$ so-potential value of $-83 \mathrm{~kJ} / \mathrm{mol}$. Reproduced from Ref. (10) with kind permission by Mary Ann Liebert, Inc. intravascular circulating half-lives in the range of minutes (11). Additional obstacles to delivery include passage across the vascular endothelium, diffusion through the extracellular matrix, and translocation across the cell membrane. Finally, oligonucleotides are associated with immune activation, further limiting their clinical application $(12,13)$.

These issues could be addressed by conjugating, complexing, or encapsulating the oligos to nanocarriers. These can be classified into three types: lipid-based, polymer-based, and inorganic nanoparticles. Nanocarrier design is optimized in terms of physicochemical properties (surface coating and particle size) in order to achieve optimal biodistribution and pharmacokinetics, to shield the oligo from degradation, to facilitate cellular uptake, and release the oligo in the proper intracellular compartment, and to satisfy requirements related to their physiological safety, including lack of immunogenicity, non-coagulation with serum proteins, and low non-specific uptake by normal tissues or cells.

As stated in Ref. (14), a major prerequisite for successful oligo delivery is the presence of positive charges on the surface of the nanoparticles that would facilitate uptake across cell membranes. However, cationic surface charges increase the chances of aggregate formation with plasma proteins. This can be overcome by the introduction of PEG on the surface of the nanocarrier. This can neutralize the surface charge and avoid interaction with plasma proteins, resulting in longer circulation half-life and faster diffusion across the extracellular matrix (15). However, this modification interferes with cellular uptake and necessitates an additional moiety for receptor-mediated endocytosis, which significantly limits the modular potential of the construct.

With respect to nanoparticle size, we are limited by the requirement that the size of the nanocarrier-oligo construct should be larger than the pore size of the glomerular filtering system $(>7 \mathrm{~nm})$ to avoid renal clearance but small enough $(<100 \mathrm{~nm})$ to avoid rapid phagocytosis by cells of the reticuloendothelial system (16-19). Typically, nanocarriers in the range of 10-100 nm in diameter are optimal to allow delivery to tissues through the enhanced permeation and retention effect. This is particularly relevant for solid tumors that are characterized by leaky vasculature $(16,20,21)$. Even in poorly permeable tumors, nanocarriers smaller than $50 \mathrm{~nm}$ can penetrate the capillary endothelium, whereas micellar nanoparticles of $70 \mathrm{~nm}$ are retained in the vasculature (22). Based on these observations, the optimal size of nanocarriers for cancer treatment can be narrowed to $10-50 \mathrm{~nm}$.

These requirements, as well as detailed descriptions of polymeric nanoparticles, lipid-based nanoparticles, and inorganic nanoparticles for oligo delivery can be found in Ref. (14). Here, we will highlight a few investigations that relate to the advancement of modularity in nanocarrier design. An ingenious new method was described earlier this year (23) that would permit the high throughput in vivo discovery of targeted nucleic acid nanotherapeutics. This methodology directly addresses the fact that nucleic acid therapeutics are limited by inefficient delivery to target tissues and by an incomplete understanding of how nanoparticle structure affects biodistribution. The authors developed a method to simultaneously measure the biodistribution of many chemically distinct nanoparticles by formulating the nanoparticles to carry specific DNA barcode oligonucleotides. These barcoded 
nanoparticles were then administered to mice in vivo. After a pre-selected time, nanoparticle biodistribution was quantified by deep sequencing the barcodes (Figure 2). This method permitted the accurate measurement of relative quantities of nucleic acid delivered to tissues and identified chemical properties promoting nanoparticle delivery to specific tissues (23). Using this system, it should be possible to rapidly select nanoparticles targeting specific organs following in vivo administration. The approach would also facilitate a deeper understanding of how the chemical structure of nanoparticles affects their delivery in vivo.

To illustrate the modular potential of nanotechnology, we focus on a recent publication by Robert Siegwart's group at the University of Texas Southwestern Medical Center (24). In their study, the authors synthesized more than 1,500 siRNAincorporating modular dendrimers using sequential, orthogonal reactions where ester degradability was systematically integrated with chemically diversified cores, peripheries, and generations (Figure 3). They then screened these dendrimers in cell lines and in mice to identify candidates that achieve high potency to tumors and low hepatotoxicity and provide a pronounced survival benefit in an aggressive liver cancer model (24). This study is just an example of the power of nanotechnology for modularity. However, multiple nanocarriers, beyond dendrimers, could be synthesized using a modular approach. These include soft/hollow-core nanoparticles, such as lipid nanoparticles, polymeric nanoparticles, carbon nanotubes, and porous silica nanoparticles, as well as hard-core nanoparticles, such as quantum dots, gold nanoparticles, and iron oxide nanoparticles. Also, modular building blocks can be utilized for the optimization of nanocarrier structure in terms of size, surface charge, spacer length, and functional groups to ensure long-term stability, the enhanced interaction with target transcriptomes, and optimal circulation half-life/biodistribution.

\section{IMAGING}

The role of imaging in cancer therapy is well established. As part of their routine work-up, patients are imaged by ultrasonography, computerized tomography (CT), MRI, or positron emission tomography (PET) to both diagnose and stage the disease, and to monitor the progress of treatment. The value and necessity for this type of analysis is indisputable. In addition to imaging, a wide variety of circulating biomarkers, such as circulating tumor cells, exosomes, and circulating cell-free DNA/RNA, are finding application in recent years. Finally, more invasive in situ diagnostic methods that involve biopsy are widely used and largely considered as the gold standard diagnostic approach.

In this review, we focus on imaging not for the diagnosis, staging, and monitoring of cancer, but rather for the specific measurement of drug delivery to the target tissue and/or the overall pharmacokinetics of the drug. Even though this kind of studies are routinely involved in the process of drug development, they are not part of patient care. Since the overall goal is the modular development of strictly personalized therapeutic agents, in the treatment paradigm that we illustrate here, knowledge about pharmacokinetics and drug delivery need to be obtained for each patient. Hopefully, this could be accomplished concurrently with the assessment of therapeutic response.
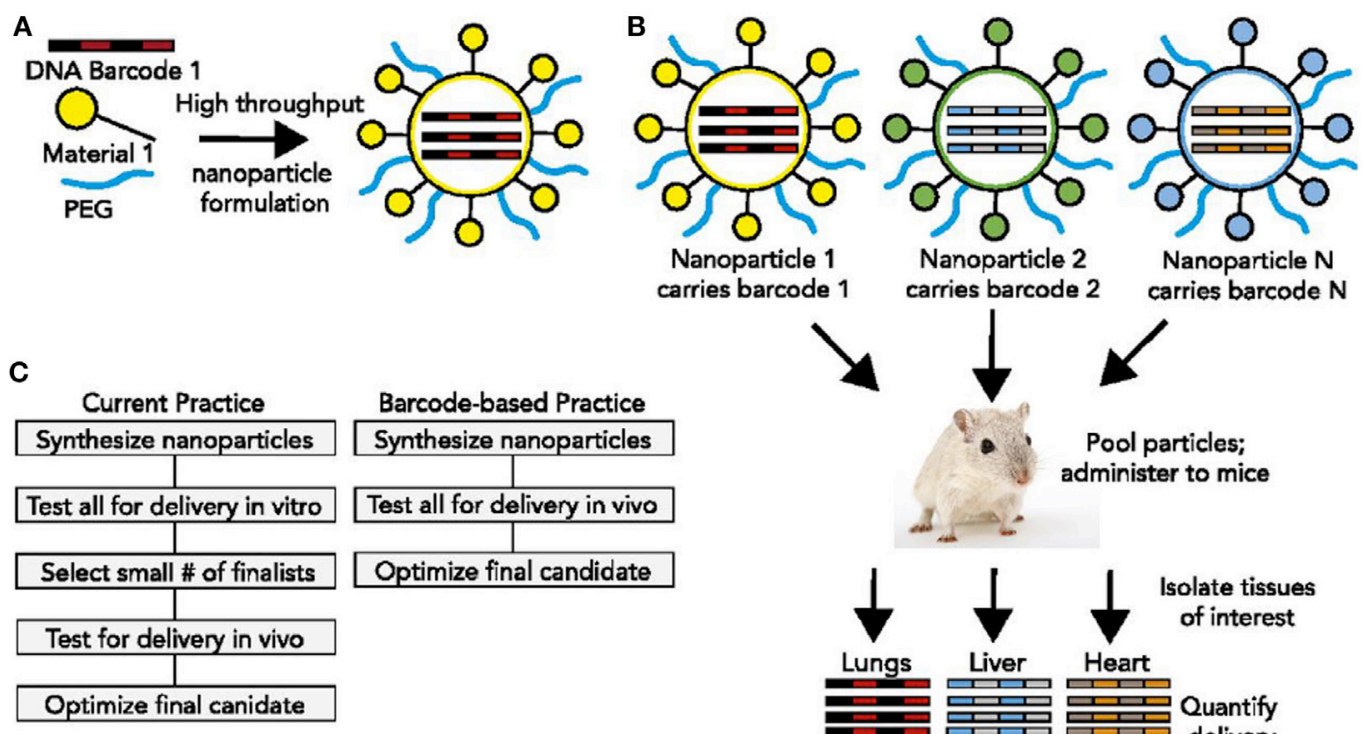

Pool particles; administer to mice

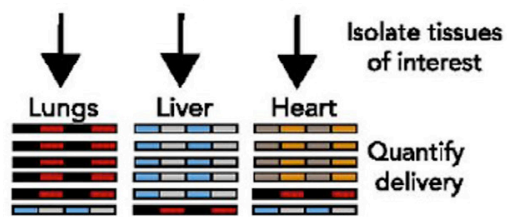

FIGURE 2 | DNA barcoded nanoparticles for high-throughput in vivo nanoparticle delivery. (A) Using high-throughput fluidic mixing, nanoparticles are formulated to carry a DNA barcode. (B) Many nanoparticles can be formulated in a single day; each nanoparticle chemical structure carries a distinct barcode. Particles are then combined and administered simultaneously to mice. Tissues are then isolated, and delivery is quantified by sequencing the barcodes. In this example, nanoparticle 1 delivers to the lungs, nanoparticle 2 delivers to the liver, and nanoparticle $N$ delivers to the heart. (C) This DNA barcode system enables multiplexed nanoparticletargeting studies in vivo, improving upon the current practice, which relies on in vitro nanoparticle screening to identify lead candidates. Reproduced from Ref. (23) with kind permission by the National Academy of Sciences. 


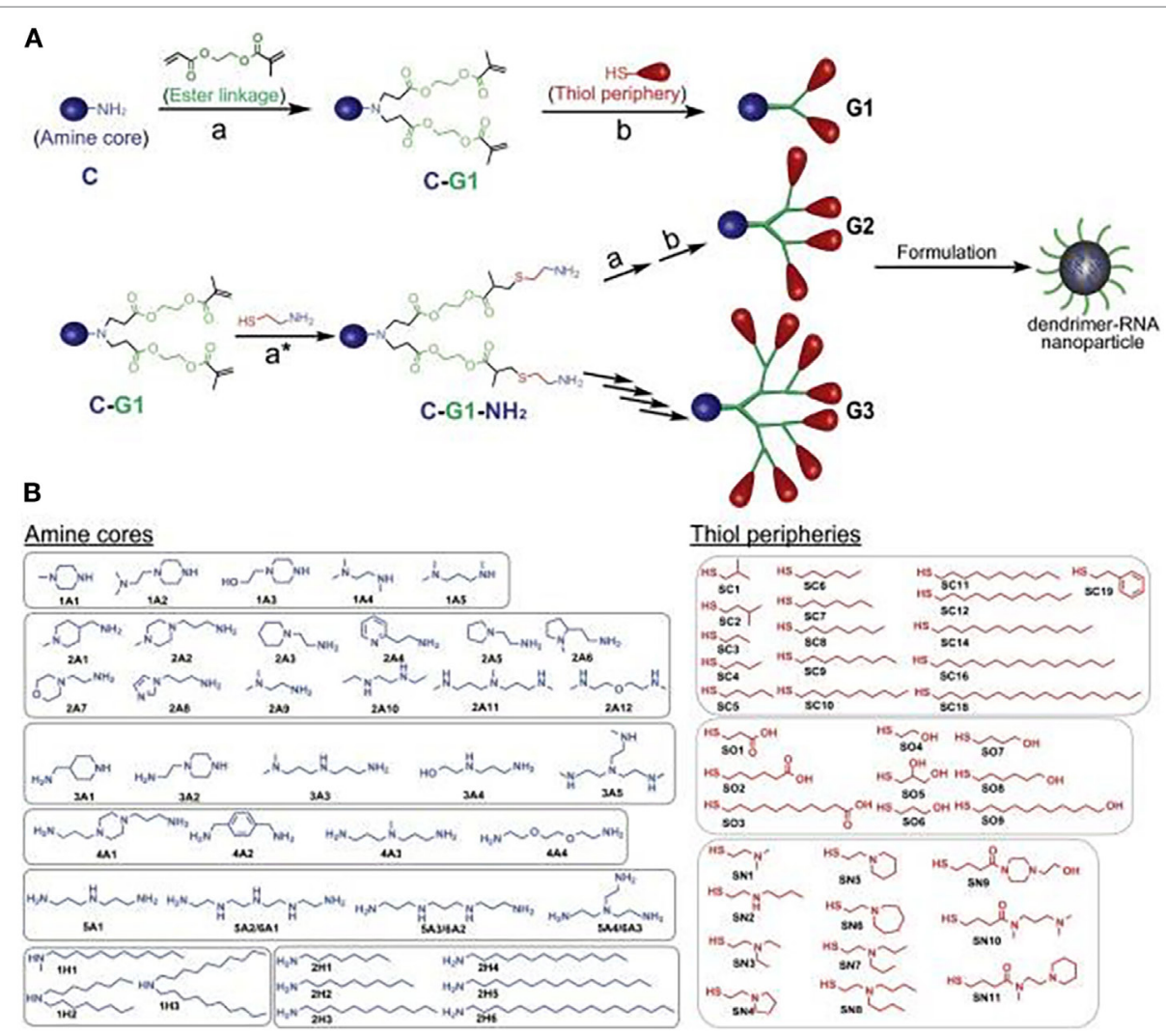

FIGURE 3 | A modular strategy for diversifying the chemical functionality and size of ester-based dendrimers allowed discovery of potent and nontoxic dendrimers for in vivo small-RNA delivery to tumor cells. (A) Orthogonal reactions accelerated the synthesis of $>1,500$ modular degradable dendrimers by combination of 42 cores $(C)$ and 36 peripheries $(P)$ through degradable linkages $(L)$ and generations. The library was established via sequential reactions. First, amines $(C)$ with a series of $\mathrm{N}-\mathrm{H}$ bonds reacted quantitatively and selectively with the less steric acrylate groups of AEMA (L). The products (C-L) then quantitatively reacted with various thiols (P) under optimized DMPP-catalyzed conditions. (B) Dendrimers were independently modulated with chemically diverse amines and thiols. Selected amines were divided into two categories: ionizable amines ( $1 \mathrm{~A}-6 \mathrm{~A})$ to tune RNA binding from $\mathrm{C}$ that generated one to six branched dendrimers, and alkyl amines $(1 \mathrm{H}-2 \mathrm{H})$ to tune NP C stabilization. Alkyl thiols (SC1-SC19) and alcohol/carboxylic acid terminated thiols (SO1-SO9) were selected to tune NP P stabilization. Aminothiols (SN1-SN11) were selected to tune P RNA binding. G2-G4 higher generation dendrimers with multiple branches were also synthesized using generation expansion reactions. Reproduced from Ref. (24) with kind permission by the National Academy of Sciences.

One study that illustrates this vision comes from Zaver Bhujwalla's group at Johns Hopkins University (25). The authors developed a PSMA-targeted nanoplex platform for theranostic imaging of prostate cancer. The therapeutic nanoplex was designed to deliver siRNA against choline kinase (Chk) along with a prodrug (5-fluorocytosine; 5-FC). Importantly, the nanoplex contained multimodal imaging reporters that permitted the assessment of its accumulation in tumors and its therapeutic effect manifested as siRNA-mediated down-regulation of Chk and the conversion of the prodrug 5-FC to the cytotoxic drug 5-FU. Specifically, SPECT/CT of mice bearing PC3-PIP and PC3-Flu tumors revealed a significantly higher uptake of the targeted nanoplex in PSMA-overexpressing PC3-PIP tumors compared to PC3-Flu tumors (Figure 4A). To assess the efficacy of siRNA-Chk to downregulate Chk, the authors acquired in vivo $1 \mathrm{H}$ MRSI of PC3-PIP tumors $48 \mathrm{~h}$ after administration of the nanoplex. As shown in Figure 4B, there was a significant decrease of the total choline signal that consists of free choline, PC, and glycerophosphocholine. Moreover, by performing 19F MRS, the authors observed the activity of the prodrug enzyme bCD, as it converted the prodrug 5-FC to 5-FU (Figure 4C) (25).

In our own work, we have strived to incorporate imaging of drug delivery as an indispensable companion to drug development. We have extensively used dextran coated iron oxide nanoparticles as delivery vehicles for siRNA or antagomir therapeutics to tumors (26-31).

Since these nanoparticles are easily detected by MRI, we have utilized this modality to measure drug bioavailability in vivo.

A specific application is illustrated in Ref. (29). The study described the testing of an imaging-capable nanodrug that was designed to inhibit the pro-metastatic miRNA-10b. The nanodrug's physicochemistry was specifically optimized to reach primary and metastatic tumor cells. Therapy was delivered to a mouse model of metastatic breast cancer. During the course of treatment, the animals were imaged by MRI in an attempt to finetune the administration schedule and design of the nanodrug. MRI revealed that the nanodrug was successfully delivered to the tumors, as shown by the decrease in transverse relaxation time 

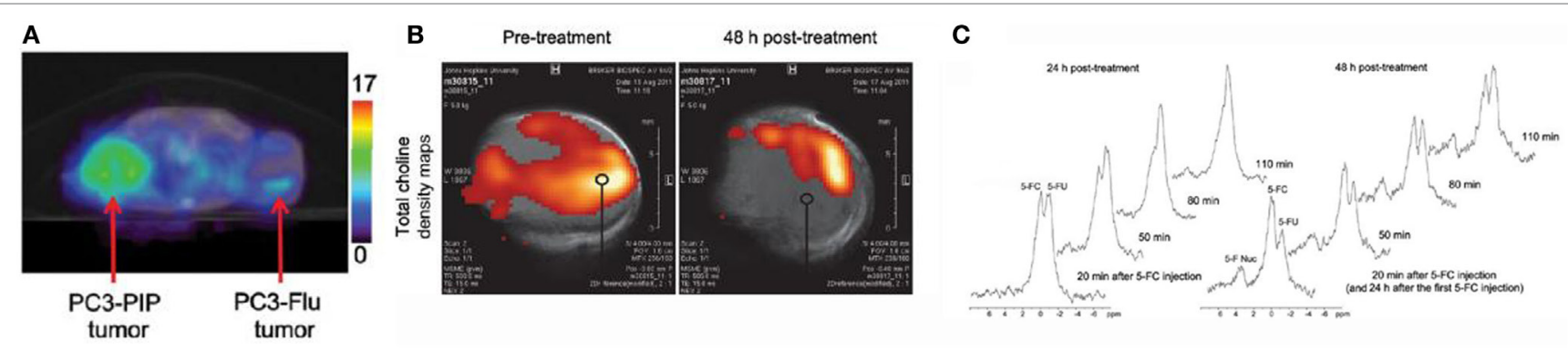

FIGURE 4 | A multimodal approach for the non-invasive assessment of drug bioavailability and therapeutic effect in a model of prostate cancer. (A) Representative SPECT images of a SCID mouse bearing PC3-PIP and PC3-Flu tumors. (B) In vivo total choline density maps from 2D CSI datasets acquired from a representative PC3-PIPtumor $\left(\sim 400 \mathrm{~mm}^{3}\right)$ before and $48 \mathrm{~h}$ after i.v. injection of the PSMA-targeted nanoplex 1 (150 mg/kg). (C) In vivo ${ }^{19} \mathrm{FMR}$ spectra acquired from a PC3-PIP tumor $\left(400 \mathrm{~mm}^{3}\right)$ at 24 and $48 \mathrm{~h}$ after i.v. injection of the PSMA-targeted nanoplex (150 mg/kg) carrying bCD and siRNA-Chk. Reproduced from Ref. (25) with kind permission by the American Chemical Society.

(Figure 5A). Quantitative analysis suggested a tendency toward nanodrug build-up in the tissue after the second treatment session (Figure 5B). These results illustrate a potentially useful application of imaging, especially when the delivery vehicle is innately imaging capable, as is the case for iron oxide nanoparticles. Such capabilities could be essential when optimizing a modular nanocarrier of this kind (29).

When the nanocarrier is not innately imaging-capable, it is highly beneficial to apply protocols for modular labeling of the nanocarrier with a radionuclide for nuclear imaging. This approach is illustrated in a study by Thomas Reiner's group at Memorial Sloan-Kettering Cancer Center (32). The authors developed an ${ }^{89} \mathrm{Zr}$-based labeling strategy for liposomal nanoparticles that accumulate in tumors via passive targeting mechanisms. Labeling was accomplished either by click labeling (CLL) or surface chelation (SCL), illustrating the versatility of the labeling process. PET/CT imaging of the radiolabeled nanoparticles was performed on a mouse model of breast cancer. Figure $6 \mathrm{~A}$ shows a comparative ${ }^{89} \mathrm{Zr}$ activity biodistribution in selected tissues after intravenous administration of the liposomes in mice bearing breast cancer xenografts. PET/CT imaging with ${ }^{89} \mathrm{Zr}$-CLL at $2 \mathrm{~h}$ after injection showed predominantly liver and spleen uptake. In contrast, ${ }^{89} \mathrm{Zr}$-SCL PET images at first showed high blood-pool activity and strong signals from liver and spleen. At $24 \mathrm{~h}$, the blood-pool signal was moderate but tumor accumulation was higher (Figure 6B) (32).

The highlighted papers represent just a first step in the direction of integrating imaging more closely with the process of drug development and treatment planning. Given the need to obtain concurrent information of therapeutic response and drug delivery, it is likely that such approaches would involve a dual-modality capability, most likely PET-MR or PET-CT. The combination of these modalities is uniquely poised to obtain quantitative co-registered information about tumor dynamics, as a function of drug accumulation and could provide clues about the failure of some therapies in a given patient from the standpoint of drug delivery. On a broader scale, the introduction of this methodology would greatly enrich the process of drug development, which is currently hampered by a limited understanding of patient-to-patient variability in terms of drug delivery.

\section{FUTURE OUTLOOK}

While exciting, the vision we outline may be criticized for being unrealistic. A major impediment to the fulfillment of this vision would likely be cost. It does not seem cost-effective to completely elucidate the genetic profile of a given patient's tumor, identify causative therapeutic targets, and design oligonucleotide probes that would normalize the tumor cell phenotype. It would also not be cost-effective to diagnose and monitor each patient using sophisticated imaging modalities at multiple time points during therapy. Finally, the cost of synthesizing individualized drugs may seem overwhelming. However, given the high degree of trial-and-error empiricism that defines the current approaches, it is likely that the cost of multiple ineffective treatments is equally great not only in terms of money but, more importantly, in terms of patient distress and treatment failure.

An additional important point is the clear need for technological advancement before the vision of fully rational therapeutics could be fulfilled. Areas that need development include the following:

1. Complete elucidation of the genetic/epigenetic regulatory pathways that lead to cancer, so that we functionally understand the transcriptome of the cancer cell. This will allow us to predict the complete array of phenotypic effects of targeting a given gene/mRNA/or miRNA;

2. Design and validation of oligonucleotide probes that have the highest specificity and no off-target effects using nonempirical computational approaches;

3. Design and optimization of delivery vehicles for these oligonucleotide probes based on target organ, desired circulation half-life, need for no systemic toxicity, etc;

4. Development of standardized labeling protocols for image guidance and the implementation of optimal imaging protocols and instrumentation for a given modality.

Despite these issues, the vision of fully rational cancer therapy inspires hope defined mostly by the promise of better outcomes. Cancer is still largely an unaddressed health issue. 

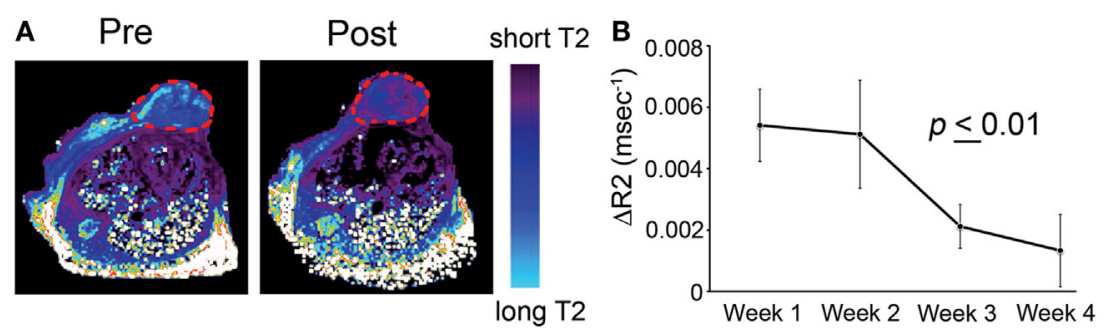

FIGURE 5 | T2-weighted magnetic resonance imaging of MN-anti-miR10b accumulation in orthotopic MDA-MB-231-luc-D3H2LN tumors. (A) Representative color-coded T2 maps before (left) and $24 \mathrm{~h}$ after (right) MN-anti-miR10b injection demonstrating a shortening of the T2 relaxation times of the tumors (outlined) consistent with nanodrug accumulation. (B) Quantitative analysis of $\Delta \mathrm{R} 2$ relaxation rates (1/T2 pre $-1 / \mathrm{T} 2$ post, ms) of the tumors, suggesting a tendency toward build-up of the MN-anti-miR10b ( $p \leq 0.01, n=12$ ). Data are represented as mean \pm SD. Reproduced from Ref. (29) with kind permission by Nature Publishing Group.

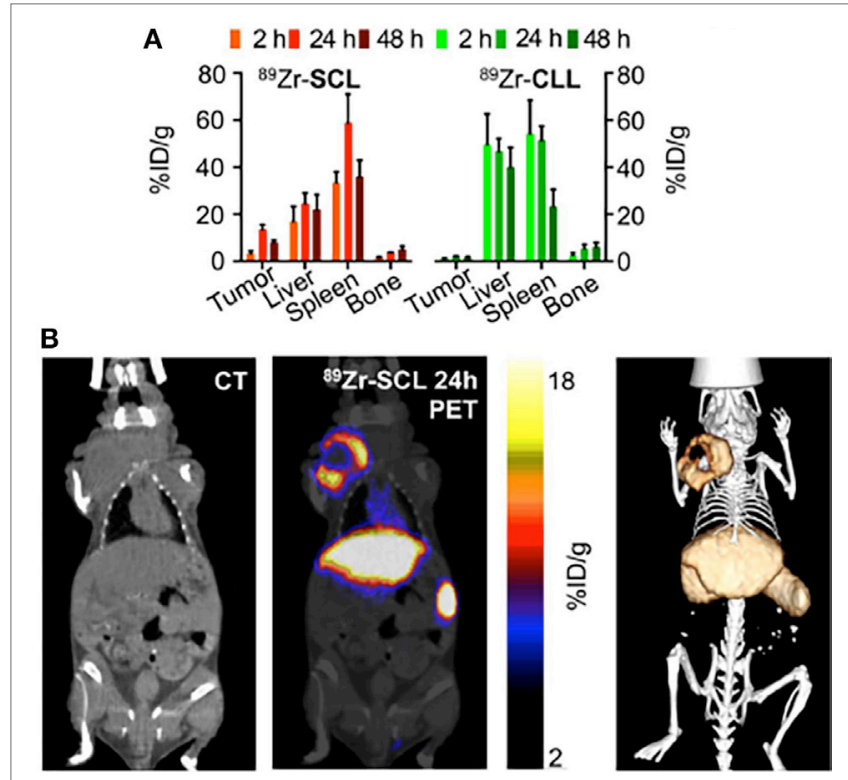

FIGURE 6 | Modular labeling for in vivo positron emission tomography (PET) imaging of tumor-homing nanoparticles. (A) Radioactivity distribution in selected tissues of ${ }^{89} \mathrm{Zr}$-SCL and ${ }^{89} \mathrm{Zr}$-CLL. (B) PET/computerized tomography (CT) imaging of ${ }^{89} \mathrm{Zr}$-SCL: CT only (left), PET/CT fusion (middle), and 3-dimensional rendering of PET/CT fusion (right) at $24 \mathrm{~h}$ after injection. Reproduced from Ref. (32) with kind permission by the Society of Nuclear Medicine and Molecular Imaging.

\section{REFERENCES}

1. Bennett CF, Swayze EE. RNA targeting therapeutics: molecular mechanisms of antisense oligonucleotides as a therapeutic platform. Annu Rev Pharmacol Toxicol (2010) 50:259-93. doi:10.1146/annurev.pharmtox.010909. 105654

2. Stein CA, Hansen JB, Lai J, Wu S, Voskresenskiy A, Hog A, et al. Efficient gene silencing by delivery of locked nucleic acid antisense oligonucleotides, unassisted by transfection reagents. Nucleic Acids Res (2010) 38(1):e3. doi:10.1093/ nar/gkp841

3. Kibler-Herzog L, Zon G, Uznanski B, Whittier G, Wilson WD. Duplex stabilities of phosphorothioate, methylphosphonate, and RNA analogs of two DNA 14-mers. Nucleic Acids Res (1991) 19(11):2979-86. doi:10.1093/ nar/19.11.2979
It remains the second most common cause of death in the US, accounting for nearly one of every four deaths. As an example, pancreatic cancer is a devastating diagnosis defined by a mere $2 \% 5$-year survival when diagnosed at an advanced inoperable stage, which defines $80 \%$ of the cases. Despite overall progress in research, the prognosis for people with pancreatic cancer has not improved in over 40 years. In light of the tremendous suffering caused by this disease and the modest progress achieved using standard treatments, it is clear that we need to explore radical, transformative approaches for therapy. The vision of fully rational drug design represents a hopeful step in that direction.

\section{AUTHOR CONTRIBUTIONS}

$\mathrm{ZM}$ conceived of the ideas presented and drafted the review. $\mathrm{BY}$ and $\mathrm{A}-\mathrm{MB}$ drafted the sections detailing the highlighted research papers and provided feedback on the discussion.

\section{FUNDING}

The study was supported under grant R01CA16346101A1 from the National Cancer Institute to ZM. A-MB was supported by the CaNCURE program. CaNCURE is a partnership between Northeastern University and Dana-Farber/Harvard Cancer Center funded by the National Cancer Institute.

4. Majlessi M, Nelson NC, Becker MM. Advantages of 2'-O-methyl oligoribonucleotide probes for detecting RNA targets. Nucleic Acids Res (1998) 26(9):2224-9. doi:10.1093/nar/26.9.2224

5. Rettig GR, Behlke MA. Progress toward in vivo use of siRNAs-II. Mol Ther (2012) 20(3):483-512. doi:10.1038/mt.2011.263

6. Koshkin AA, Wengel J. Synthesis of novel 2',3'-linked bicyclic thymine ribonucleosides. J Org Chem (1998) 63(8):2778-81. doi:10.1021/jo972239c

7. Fontenete S, Barros J, Madureira P, Figueiredo C, Wengel J, Azevedo NF. Mismatch discrimination in fluorescent in situ hybridization using different types of nucleic acids. Appl Microbiol Biotechnol (2015) 99(9):3961-9. doi:10.1007/ s00253-015-6389-4

8. Deleavey GF, Damha MJ. Designing chemically modified oligonucleotides for targeted gene silencing. Chem Biol (2012) 19(8):937-54. doi:10.1016/j. chembiol.2012.07.011 
9. Khvorova A, Watts JK. The chemical evolution of oligonucleotide therapies of clinical utility. Nat Biotechnol (2017) 35(3):238-48. doi:10.1038/ nbt. 3765

10. Koch T, Shim I, Lindow M, Orum H, Bohr HG. Quantum mechanical studies of DNA and LNA. Nucleic Acid Ther (2014) 24(2):139-48. doi:10.1089/ nat.2013.0465

11. Soutschek J, Akinc A, Bramlage B, Charisse K, Constien R, Donoghue M, et al. Therapeutic silencing of an endogenous gene by systemic administration of modified siRNAs. Nature (2004) 432(7014):173-8. doi:10.1038/ nature 03121

12. Judge AD, Sood V, Shaw JR, Fang D, McClintock K, MacLachlan I. Sequencedependent stimulation of the mammalian innate immune response by synthetic siRNA. Nat Biotechnol (2005) 23(4):457-62. doi:10.1038/nbt1081

13. Hornung V, Guenthner-Biller M, Bourquin C, Ablasser A, Schlee M, Uematsu S, et al. Sequence-specific potent induction of IFN- $\alpha$ by short interfering RNA in plasmacytoid dendritic cells through TLR7. Nat Med (2005) 11(3):263-70. doi: $10.1038 / \mathrm{nm} 1191$

14. Yoo B, Medarova Z. Nanoformulations for pharmacological siRNA delivery in cancer. In: Bulte J, Modo M, editors. Design and Applications of Nanoparticles in Biomedical Imaging. Switzerland: Springer International Publishing (2017). p. 171-86.

15. Stylianopoulos T, Poh M-Z, Insin N, Bawendi MG, Fukumura D, Munn LL, et al. Diffusion of particles in the extracellular matrix: the effect of repulsive electrostatic interactions. Biophys J (2010) 99(5):1342-9. doi:10.1016/j. bpj.2010.06.016

16. Lin Q, Chen J, Zhang Z, Zheng G. Lipid-based nanoparticles in the systemic delivery of siRNA. Nanomedicine (2014) 9(1):105-20. doi:10.2217/ nnm.13.192

17. Kanasty RL, Whitehead KA, Vegas AJ, Anderson DG. Action and reaction: the biological response to siRNA and its delivery vehicles. Mol Ther (2012) 20(3):513-24. doi:10.1038/mt.2011.294

18. Aird WC. Phenotypic heterogeneity of the endothelium I. Structure, function, and mechanisms. Circ Res (2007) 100(2):158-73. doi:10.1161/01. RES.0000255691.76142.4a

19. Wisse E, Jacobs F, Topal B, Frederik P, De Geest B. The size of endothelial fenestrae in human liver sinusoids: implications for hepatocyte-directed gene transfer. Gene Ther (2008) 15(17):1193-9. doi:10.1038/gt.2008.60

20. Choi HS, Liu W, Misra P, Tanaka E, Zimmer JP, Ipe BI, et al. Renal clearance of quantum dots. Nat Biotechnol (2007) 25(10):1165-70. doi:10.1038/nbt1340

21. Singh S, Sharma A, Robertson GP. Realizing the clinical potential of cancer nanotechnology by minimizing toxicologic and targeted delivery concerns. Cancer Res (2012) 72(22):5663-8. doi:10.1158/0008-5472.CAN-12-1527

22. Cabral H, Matsumoto Y, Mizuno K, Chen Q, Murakami M, Kimura M, et al. Accumulation of sub-100 nm polymeric micelles in poorly permeable tumours depends on size. Nat Nanotechnol (2011) 6(12):815-23. doi:10.1038/ nnano.2011.166
23. Dahlman JE, Kauffman KJ, Xing Y, Shaw TE, Mir FF, Dlott CC, et al. Barcoded nanoparticles for high throughput in vivo discovery of targeted therapeutics. Proc Natl Acad Sci U S A (2017) 114(8):2060-5. doi:10.1073/pnas. 1620874114

24. Zhou K, Nguyen LH, Miller JB, Yan Y, Kos P, Xiong H, et al. Modular degradable dendrimers enable small RNAs to extend survival in an aggressive liver cancer model. Proc Natl Acad Sci U S A (2016) 113(3):520-5. doi:10.1073/ pnas. 1520756113

25. Chen Z, Penet MF, Nimmagadda S, Li C, Banerjee SR, Winnard PT Jr, et al. PSMA-targeted theranostic nanoplex for prostate cancer therapy. ACS Nano (2012) 6(9):7752-62. doi:10.1021/nn301725w

26. Ghosh SK, Yigit MV, Uchida M, Ross AW, Barteneva N, Moore A, et al. Sequence-dependent combination therapy with doxorubicin and a survivinspecific small interfering RNA nanodrug demonstrates efficacy in models of adenocarcinoma. Int J Cancer (2014) 134(7):1758-66. doi:10.1002/ijc.28499

27. Medarova Z, Pham W, Farrar C, Petkova V, Moore A. In vivo imaging of siRNA delivery and silencing in tumors. Nat Med (2007) 13(3):372-7. doi:10.1038/ $\mathrm{nm} 1486$

28. Kumar M, Yigit M, Dai G, Moore A, Medarova Z. Image-guided breast tumor therapy using a small interfering RNA nanodrug. Cancer Res (2010) 70(19):7553-61. doi:10.1158/0008-5472.CAN-10-2070

29. Yigit MV, Ghosh SK, Kumar M, Petkova V, Kavishwar A, Moore A, et al. Context-dependent differences in miR-10b breast oncogenesis can be targeted for the prevention and arrest of lymph node metastasis. Oncogene (2013) 32(12):1530-8. doi:10.1038/onc.2012.173

30. Yoo B, Kavishwar A, Ross A, Wang P, Tabassum DP, Polyak K, et al. Combining miR-10b-targeted nanotherapy with low-dose doxorubicin elicits durable regressions of metastatic breast cancer. Cancer Res (2015) 75(20):4407-15. doi:10.1158/0008-5472.CAN-15-0888

31. Yoo B, Kavishwar A, Wang P, Ross A, Pantazopoulos P, Dudley M, et al. Therapy targeted to the metastatic niche is effective in a model of stage IV breast cancer. Sci Rep (2017) 7:45060. doi:10.1038/srep45060

32. Perez-Medina C, Abdel-Atti D, Zhang Y, Longo VA, Irwin CP, Binderup T, et al. A modular labeling strategy for in vivo PET and near-infrared fluorescence imaging of nanoparticle tumor targeting. J Nucl Med (2014) 55(10):1706-11. doi:10.2967/jnumed.114.141861

Conflict of Interest Statement: ZM is Founder, Director, and Scientific Advisory Board Member of TransCode Therapeutics, Inc.

Copyright (C) 2017 Yoo, Billig and Medarova. This is an open-access article distributed under the terms of the Creative Commons Attribution License (CC BY). The use, distribution or reproduction in other forums is permitted, provided the original author(s) or licensor are credited and that the original publication in this journal is cited, in accordance with accepted academic practice. No use, distribution or reproduction is permitted which does not comply with these terms. 